\title{
金属硫蛋白对大鼠心肌细胞缺氧一复氧 损伤的保护作用*
}

\author{
李光萍唐朝枢 \\ (北京医科大学病理生理教研室) \\ 程 时 \\ （北京医科大学生物物理教研室）
}

\section{关链词金属硫蛋白、心肌细胞、缺氧一复氧损伤}

缺血一定时间的心肌恢复灌流后,组织损伤反而进行性加重, 出现缺血心肌的再灌注损伤 (reperfusion injury)，其发病机制还不清楚,目前尚缺少有效的防治措施。大量研究表明，心 肌缺血-再灌注损伤与缺氧一复氧损伤 (oxygen paradox)、缺钻-复钙损伤 (calcium paradox) 有相似的病理表现和发病机制,推测分子氧和铂离子在缺血一再灌注损伤的发病过程中起着重 要的作用 ${ }^{[1]}$. 金属硫蛋白 (metallothionein, MT) 是生物机体内含有多量硫和金属的非酶蛋 白,起着应激保护蛋白的作用。本工作在耐受钻的离体大鼠心肌细胞缺氧一复氧模型上, 观察 MT 的细胞保护作用,并进一步观察 MT 对自由基发生系统 (free radical generating system) 引起的大鼠心肌细胞脂质过氧化 (lipid peroxidation) 的影响, 以探讨 MT 的作用机理及 其治疗应用的可能性。

\section{一、材料和方法}

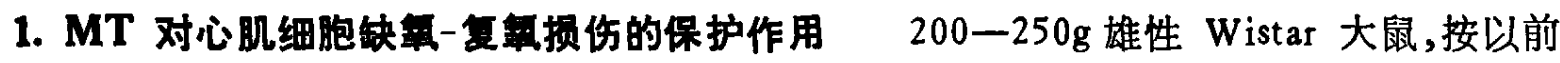
叙述的方法 ${ }^{[2]}$ 用含胶原酶 (I 型, Sigma Co $)$ 的 Krebs-Henseleit $(\mathrm{K}-\mathrm{H})$ 液灌流,分离出成年 大鼠耐受钻的心肌细胞, 悬浮于不含葡萄糖的 $\mathrm{K}-\mathrm{H}$ 液 $\left(\mathrm{Ca}^{2+} 1.25 \mathrm{mmol} / \mathrm{l}\right)$, 按下述的分组 分置于硅化过的小试管中 $\left(2 \mathrm{ml} /\right.$ 管), 在恒温水浴振荡器上 $37^{\circ} \mathrm{C}$ 䁔育。（1) 假缺氧对照： $95 \%$ $\mathrm{O}_{2}: 5 \% \mathrm{CO}_{2}$ 䁔育 $60 \mathrm{~min}$; (2) 对照: $95 \% \mathrm{~N}_{2}: 5 \% \mathrm{CO}_{2}$ 缺氧瞹育 $40 \mathrm{~min}$, 再换 $95 \% \mathrm{O}_{2}: 5 \% \mathrm{CO}^{2}$

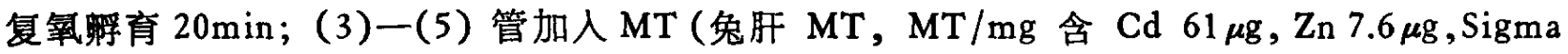
Co）终浓度分别为 $10^{-9}, 10^{-6}, 10^{-7} \mathrm{~mol} / 1$, 睬育方法与对炤组相同。6 次实验, 每次做两平行 管. 结束稃育后，用台盘蓝排斥试验在光镜下检测杆形细胞存活率; 考马斯亮蓝法蛋白定量; 一半细胞用硫代巴比妥酸法测定细胞脂质过氧化产物丙二醛 (MDA) 含量, 另一半细胞经消 化处理后, 在原子吸收分光光度计 (GGX-1B 型)上测定细胞钙含量.

\section{MT 对心悓细胞脂质过策化的影响四例大鼠心脏按上述方法分离出心肌细胞, 分}

本文1988 年 6 月 4 日收到. 1988 年 8 月24日收到修改稿.

“国家“七五”攻关资助项目. 
别置于 5 组试管中 (2ml/管):（1)空白对照：仅加人 $\mathrm{K}-\mathrm{H}$ 缓冲液; (2)对照：加人 $\mathrm{FeCl}_{2}$ 和 抗坏血酸作为自由基发生系统, 终浓度分别为 0.1 和 $0.5 \mathrm{mmol} / 1$; (3)-(5) 管除加人自由基 发生系统外, 还加入 $\mathrm{MT}$ 分别为 $2 \times 10^{-5}, 2 \times 10^{-6}, 2 \times 10^{-7} \mathrm{~mol} / 1$. 在恒温水浴振荡器上 $\left(37{ }^{\circ} \mathrm{C}\right), 95 \% \mathrm{O}_{2}: 5 \% \mathrm{CO}_{2}$ 門育 $20 \mathrm{~min}$, 然后分别测定各管的杆形细胞存活率和 $\mathrm{MDA}$ 含量.

实验结果用均值士标准误差表示, 配对资料 $\iota$ 检验作统计学处理。

\section{二、结 果}

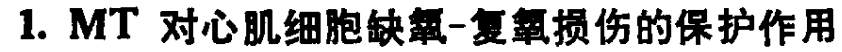

实验分离的大鼠心肌细胞在含钙 1.25 $\mathrm{mmol} / 1$ 的培养液里， $90 \%$ 以上排斥台盘蓝紫色， $70 \%$ 以上呈杆型。心肌细胞缺氧一复氧损 伤(对照)表现为细胞孪缩, 结合染料, 杆形细胞存活率显著减少, 而细胞脂质过氧化产物 MDA 和细胞内钙含量明显增加. $\mathrm{MT}$ 处理有效地保护心肌细胞抵抗其缺氧一复氧损伤, 与对照组 相比较, 杆形细胞存活率较高, 而细胞 $\mathrm{MDA}$ 和 $\mathrm{Ca}^{2+}$ 含量较低. 其保护作用呈剂量依赖性 (风表 1).

表 $1 \mathrm{MT}$ 对心肌细胞缺氧一复氧损伤的影响

\begin{tabular}{|c|c|c|c|c|c|}
\hline & \multirow{2}{*}{ 假缺軍 } & \multirow{2}{*}{ 对照 } & \multicolumn{3}{|c|}{$\mathrm{MT}(\mathrm{mol} / \mathrm{l})$} \\
\hline & & & $10^{-5}$ & $10^{-6}$ & $10^{-7}$ \\
\hline 细胞存活率(\%) & $73.2 \pm 2.1^{* *}$ & $56.4 \pm 2.1$ & $64.9 \pm 1.4^{* *}$ & $60.3 \pm 1.5 *$ & $56.4 \pm 1.9$ \\
\hline MDA含量( $\mathrm{nmol} / \mathrm{mg}$ 蛋白) & $0.40 \pm 0.04 * *$ & $0.79 \pm 0.06$ & $0.55 \pm 0.04 * *$ & $0.72 \pm 0.05^{*}$ & $0.77 \pm 0.06$ \\
\hline $\mathrm{Cs}^{2+}$ 含量 $(\mathrm{nmol} / \mathrm{mg}$ 蛋白 $)$ & $10.7 \pm 2.4 * *$ & $36.8 \pm 1.2$ & $10.7 \pm 2.5^{* *}$ & $24.9 \pm 2.5^{*}$ & $36.3 \pm 0.7$ \\
\hline
\end{tabular}

$n=6$, 与对照组相比较: ${ }^{*} P<0.05 ;{ }^{*} P<0.01$.

2. MT 对心肌细胞脂质过氧化的影响 自由基发生系统引起心肌细胞 MDA 含量 成 倍增加,杆形细胞存活率显著降低. $\mathrm{MT}$ 明显拮抗自由基发生系统引起的细胞损伤, 与对照 组相比较, 和 MT 共同卯化的心肌细胞的 MDA 含量较低, 而细胞存活率高, 其效应呈剂量 依赖性 (表 2)。

表 $2 \mathrm{MT}$ 对心肌细胞脂质过氧化的影响

\begin{tabular}{|c|c|c|c|c|c|}
\hline & \multirow{2}{*}{ 空 白 } & \multirow{2}{*}{ 对 盟 } & \multicolumn{3}{|c|}{ MT $(\mathrm{mol} / \mathrm{l})$} \\
\hline & & & $2 \times 10^{-3}$ & $2 \times 10^{-6}$ & $2 \times 10^{-1}$ \\
\hline 细㔠存活率(\%) & $72.8 \pm 0.7 * *$ & $56.4 \pm 3.6$ & $66.4 \pm 1.7^{*}$ & $62.1 \pm 2.8^{*}$ & $57.8 \pm 2.1$ \\
\hline MDA含量 $(\mathrm{nmol} / \mathrm{mg}$ 蛋白) & $0.45 \pm 0.04 * *$ & $0.94 \pm 0.07$ & $0.76 \pm 0.08 * *$ & $0.80 \pm 0.10^{*}$ & $0.84 \pm 0.14$ \\
\hline
\end{tabular}

$n=4, *$ 与 **同表1.

\section{三、讨 论}

MT 是生物体中广泛存在的富含半胱氨酸残基的低分子量蛋白（在哺乳动物其行子量是 $6100)$,分子上结合有 $\mathrm{Cd}, \mathrm{Zn}$ 等多种重金属离子, 其生理功能尚未完全阐明。近年发现在炎 症、感染、辐射及各种应激刺激时, 肝脏合成 $M T$ 增加, $M T$ 参与细胞代谢调节和应激时细胞 的适应反应, 推测是机体对外源性损伤的第一线防御机构之一 ${ }^{[3]}$. 但迄今尚无在疾病模型上. 
治疗应用的报道. 本实验在大鼠心肌细胞上,观察发现 MT 显著抑制细胞缺篻一复氧损伤,表 现为提高了细胞存活率，抑制了心肌细胞钙含量和 MDA 含量的增加。目前认为 $\mathrm{Da}^{2+}$ 内流 导致的细胞内钻超负荷是心肌缺血一再灌注损伤的重要发病环节,而微量的 $\mathrm{Cd}$ 等重金属离子 能有效地阻止细胞外钻的内流 ${ }^{[4]}$, 推测 MT 分子中的重金属离子对阻止钻超负荷的发生有重 要意义,但需进一步研究证实.

缺血心肌再灌注时产生大量活性莘，膜脂质过氧化导致膜功能与结构损伤。Thornalley 等 ${ }^{[3]}$ 报道 MT 对氧自由基,尤其是对羟自由基 (.OH) 有较强的清除作用,其作用可能与MT 分子上的半胱氨酸残基 (一 $\mathrm{SH}$ ) 有关。本实验 $\mathrm{MT}$ 显著拮抗自由基发生系统 $\left(\mathrm{FeCl}_{2}\right.$, 抗坏 血酸)引起的心肌细胞膜脂质过氧化,降低细胞 MDA 含量并提高细胞存活率, 其效应呈剂量 依赖性。实验结果提示, MT 作为有效的细胞保护剂, 对于缺血心肌再灌注损伤是很有前途 的防治药物。

MT 本身是无毒性的低分子量蛋白,但目前 MT 的提取全是采用 Cd 饱和法制备出 Cd$M T$ ，体内应用存在着 Cd 中毒的肝和肾严重损伤的问题, MT 的临床应用尚需要改革 MT 的制备方法，如用对身体无毒的 $\mathrm{Zn}$ 代替 $\mathrm{Cd}$ 制备 $\mathrm{Zn}-\mathrm{MT}$.

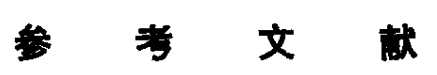

[1] Braunwald, E. and Kloner, B.A., J Clin Invest, 76(1985), 1713-1719.

[ 2] 唐朝枢等, 北京医科大学学报,19(1987), 299-301.

[3] 程时,生理科学进展, 18(1987), 352-354.

[4] Rich, T. L. and Langer, G.A., Circ Res., 51 (1982), 131-141.

[5] Thornalley, P.J. and Vasak, M, Biockim. Biophys. Acra, 827(1985), 36-44. 